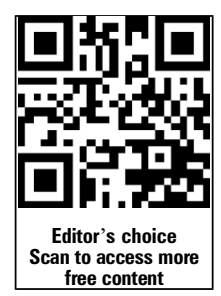

${ }^{1}$ Division of Cardiology, Department of Internal Medicine II, Medical University of Vienna, Vienna, Austria ${ }^{2}$ Division of Oncology and Hematology, Department of Internal Medicine I, Medical University of Vienna, Austria ${ }^{3}$ Division of Cardio-ThoracicVascular Anesthesia and Intensive Care Medicine, Department of Anesthesia, Medical University of Vienna, Vienna, Austria

${ }^{4}$ Complexity Research, Vienna, Austria

${ }^{5}$ Department of Gynecology, Medical University of Vienna, Vienna, Austria

${ }^{6}$ Department of Otorhinolaryngology, Head and Neck Surgery, Medical University of Vienna, Austria

\section{Correspondence to} Dr Martin Hülsmann, Division of Cardiology, Department of Internal Medicine II, Medical University of Vienna, Austria, Währinger Gürtel 18-20, 1090 Vienna, Austria; martin. huelsmann@meduniwien.ac.at

Received 20 March 2015 Revised 23 June 2015 Accepted 13 July 2015 Published Online First 28 September 2015

\section{SLinked}

- http://dx.doi.org/10.1136/ heartjnl-2015-308208

\section{CrossMark}

\footnotetext{
To cite: Pavo $N$, Raderer $M$ Hülsmann $M$, et al. Heart 2015;101:1874-1880.
}

\title{
Cardiovascular biomarkers in patients with cancer and their association with all-cause mortality
}

\author{
Noemi Pavo, ${ }^{1}$ Markus Raderer, ${ }^{2}$ Martin Hülsmann, ${ }^{1}$ Stephanie Neuhold, ${ }^{3}$ \\ Christopher Adlbrecht, ${ }^{1}$ Guido Strunk, ${ }^{4}$ Georg Goliasch, ${ }^{1}$ Heinz Gisslinger, ${ }^{2}$ \\ Günther G Steger, ${ }^{2}$ Michael Hejna, ${ }^{2}$ Wolfgang Köstler, ${ }^{2}$ Sabine Zöchbauer-Müller, ${ }^{2}$ \\ Christine Marosi, ${ }^{2}$ Gabriela Kornek, ${ }^{2}$ Leo Auerbach, ${ }^{5}$ Sven Schneider, ${ }^{6}$ \\ Bernhard Parschalk, ${ }^{6}$ Werner Scheithauer, ${ }^{2}$ Robert Pirker, ${ }^{2}$ Johannes Drach, ${ }^{2}$ \\ Christoph Zielinski, ${ }^{2}$ Richard Pacher ${ }^{1}$
}

\begin{abstract}
Objective Patients with cancer may display elevated levels of B-type natriuretic peptide (BNP) and highsensitive troponin $\mathrm{T}$ (hsTnT) without clinical manifestation of cardiac disease. This study aimed to evaluate circulating cardiovascular hormones and hsTnT and their association with mortality in cancer.
\end{abstract}

Methods We prospectively enrolled 555 consecutive patients with a primary diagnosis of cancer and without prior cardiotoxic anticancer therapy. N-terminal pro BNP (NT-proBNP), mid-regional pro-atrial natriuretic peptide (MR-proANP), mid-regional pro-adrenomedullin (MRproADM), C-terminal pro-endothelin-1 (CT-proET-1), copeptin, hsTnT, proinflammatory markers interleukin 6 (IL-6) and $C$ reactive protein (CRP), and cytokines serum amyloid A (SAA), haptoglobin and fibronectin were measured. All-cause mortality was defined as primary endpoint.

Results During a median follow-up of 25 (IQR 16-31) months, 186 (34\%) patients died. All cardiovascular hormones and hsTnT levels rose with tumour stage progression. All markers were significant predictors of mortality with HRs per IQR of $1.54(95 \% \mathrm{Cl} 1.24$ to 1.90 , $\mathrm{p}<0.001)$ for NT-proBNP, $1.40(95 \% \mathrm{Cl} 1.10$ to 1.79 , $\mathrm{p}<0.01)$ for MR-proANP, 1.31 (95\% Cl 1.19 to 1.44 , $\mathrm{p}<0.001)$ for MR-proADM, $1.21(95 \% \mathrm{Cl} 1.14$ to $1.30, p<0.001)$ for CT-proET-1, $1.22(95 \% \mathrm{Cl} 1.04$ to $1.42, p=0.014)$ for copeptin and $1.21(95 \% \mathrm{Cl} 1.13$ to $1.32, p<0.001)$ for hsTnT, independent of age, gender, tumour entity and stage, and presence of cardiac comorbidities. NT-proBNP, MR-proANP, MR-proADM and hsTnT displayed a significant correlation with IL-6 and CRP. Conclusions Circulating levels of cardiovascular peptides like NT-proBNP, MR-proANP, MR-proADM, CT-pro-ET-1 and hsTnT were elevated in an unselected population of patients with cancer prior to induction of any cardiotoxic anticancer therapy. The aforementioned markers and copeptin were strongly related to all-cause mortality, suggesting the presence of subclinical functional and morphological myocardial damage directly linked to disease progression.

\section{INTRODUCTION}

Cancer is a substantial socioeconomic burden accounting for significant morbidity and mortality in developed countries. ${ }^{1}$ Malignant cells have been shown to manipulate the endocrine system, controlling hormonal secretion while expressing the appropriate receptors to promote tumour survival and progression. ${ }^{2}$ Modifying humoral regulation is a main therapeutic modality in the current anticancer therapy for certain types of cancer. Additionally, quantifying plasma hormones has been established in routine clinical practice for tumour diagnosis and surveillance.

In terms of mortality, cancer is only outweighed by cardiovascular disease. The purely mechanistic view of cardiac disease has been extended for the last several decades, and similarly to cancer, now includes a more sophisticated perception focusing on the role of neurohumoral activation and inflammation. ${ }^{3}{ }^{4}$ Vasoactive and diuretic hormones are directly related to the severity and, more importantly, to the development and progression of cardiac disease. ${ }^{5}$ The current heart failure therapy is based on the observation that direct modulation of neurohumoral pathways results in a significant reduction of disease progression.

Recent publications reported elevated levels of several emerging cardiovascular neurohormones, which mostly derive from vascular endothelial cells, in patients with cancer. ${ }^{7-11}$ Malignant cells were shown to produce both vasoactive peptides (adrenomedullin $(\mathrm{ADM}){ }^{7} \quad$ vasopressin $(\mathrm{AVP})^{8}$ or endothelin-1 (ET-1) $\left.{ }^{9}\right)$, as well as cardiac hormones (atrial natriuretic peptide (ANP) ${ }^{12}$ and B-type natriuretic peptide $(\mathrm{BNP})^{13}$ ), yet the physiological role of the latter remains unclear. In most studies, there was no evidence for an underlying morphological cardiac substrate, and the elevated levels of natriuretic peptides have been, therefore, considered to be false-positive findings. ${ }^{11} 14$ Lately, elevated levels of troponin $\mathrm{T}(\mathrm{TnT})$ have also been detected in cancer, ${ }^{15} 16$ which have been associated with worse prognosis. ${ }^{15}$ In contrast to natriuretic hormones, which primarily represent a functional measure, TnT is a specific morphological marker for cardiac cell destruction. Similarly to BNP, increased levels of $\mathrm{TnT}$ were also found without evidence of cardiac involvement. However, the association between advanced cancer stages and cancer cachexia potentially accompanied by cardiac wasting is a well-described phenomenon, ${ }^{17}$ and heart failure 
therapy has been proven successful at improving survival in small animal studies. ${ }^{18}$ This implies that activated cardiovascular neurohormones are less innocent bystanders and equally that TnT should not be overlooked, but rather be viewed as a pathophysiologically relevant marker for estimating the risk of developing systemic cardiovascular disease.

We aimed to investigate the relationship between circulating cardiovascular peptides and mortality in an unselected population suffering from cancer in order to reveal the humoral crosstalk between cancer and cardiac disease.

\section{METHODS}

\section{Study population}

Between April 2011 and June 2013, we prospectively enrolled consecutive patients with cancer at the Vienna General Hospital, a university-affiliated tertiary care centre. Eligible patients had suspected or confirmed cancer at first presentation, and were excluded if they had received any prior cardiotoxic therapy, showed symptoms of non-stable cardiac disease or clinical signs of infection or if the diagnosis of cancer could not be confirmed in the work-up process. Patients were classified according to tumour entity and tumour stage. Traditional risk factors such as hypertension, diabetes mellitus, smoking status and medical therapy were recorded. Cardiac status was considered to be normal in the absence of cardiac disease, ECG abnormalities and N-terminal pro BNP (NT-proBNP) levels $<400 \mathrm{pg} / \mathrm{mL}$. Otherwise, we performed echocardiography to register a cardiac disorder. A significant echocardiographic finding was defined as follows: mildly, moderately or severely reduced LV or RV function, moderate or severe valvular disease or diastolic dysfunction with pseudonormal or restrictive filling patterns. Abnormal cardiac status was then defined as a history of a cardiac disease or an abnormal ECG, regardless of echocardiographic findings or a significant echocardiographic finding in patients with NT-proBNP levels $\geq 400 \mathrm{pg} / \mathrm{mL}$.

Venous blood samples were obtained at first hospital presentation and analysed on-site, according to our local laboratory standard procedures. In addition to routinely available measurements, including $\mathrm{C}$ reactive protein (CRP), haptoglobin, fibronectin, serum amyloid A (SAA), interleukin 6 (IL-6), hsTnT and NT-proBNP, also levels of mid-regional pro-ANP (MR-proANP), mid-regional pro-ADM (MR-proADM), CT-proET-1 and copeptin-the stable, but inactive fragment of the AVP prohormonewere determined.

Written, informed consent was obtained from all study participants. The study protocol complies with the Declaration of Helsinki, and was approved by the local ethics committee of the Medical University of Vienna (EK 736/2010).

\section{Assays}

CRP, haptoglobin, fibronectin and SAA levels were determined in EDTA and heparinised plasma by means of particle-enhanced immunonephelometry using the BN II System (Siemens Healthcare Diagnostics, Marburg, Germany). Serum IL-6 was detected with a specific ELISA (eBioscience, Vienna, Austria). hsTnT and NT-proBNP measurements were performed in heparin plasma using the Elecsys Systems (Roche Diagnostics, Mannheim, Germany). MR-proANP, MR-proADM, CT-proET-1 and copeptin were measured in EDTA plasma using specific sandwich immunoassays (BRAHMS, Hennigsdorf/Berlin, Germany).

\section{Study endpoint}

All-cause mortality was the primary study endpoint, and was obtained from the Central Office of Civil Registration, Austria.

\section{Statistical analysis}

Continuous data were presented as median and IQR and categorical data as counts and percentages.

Cox proportional hazard regression analysis was used to evaluate the effect of variables on the overall survival. We adjusted for established risk factors, cardiac status and renal function in a basic model, to account for potential confounding effects. The basic model was built from the variables age, gender, tumour entity (with $\mathrm{n}>5$ ) and tumour stage, which were included as possible predictors in a stepwise forward Cox regression. The $p$ value for entering the stepwise model was set to 0.05 , and the $p$ value for exclusion was set to 0.10 . To assess the robustness of the basic model and to test against overfitting, an additional bootstrap procedure was performed, repeating the variable selection for each of a total of 500 samples using the same entering and exclusion criteria. The frequency of a variable entering the model was counted. Significant predictors of the stepwise Cox regression, which entered in more than half of the bootstrap samples, were included. Finally, glomerular filtration rate (GFR) and cardiac status were built in to obtain the basic model. For all Cox regression models, the proportional hazard assumption was assessed and satisfied for all variables based on time interaction tests. For NT-proBNP, MR-proANP and copeptin logarithmic transformations were used in all regression models as they outperformed the untransformed datasets in single predictor models. Results are presented as HRs per IQR. Additionally, Harrell's concordance index (c-index) was used as a measure of the overall performance of the Cox regression models. The c-index can be interpreted similarly to the wellknown area under the curve of the receiver operating characteristics (ROC) curve.

For further analysis of the effects of NT-proBNP, MR-proANP, MR-proADM and hsTnT on mortality, the variables were dichotomised at selected cut-off values (either upper limit of the normal range or median if normal values were not applicable): $125 \mathrm{pg} / \mathrm{mL}$ for NT-proBNP, $0.49 \mathrm{nmol} / \mathrm{L}$ for MR-proADM, $66.6 \mathrm{pnmol} / \mathrm{L}$ for CT-proET-1 and $0.005 \mathrm{ng} / \mathrm{mL}$ for hsTnT. Time-to-event analysis was performed for the datasets by the means of a log-rank test. The cumulative probabilities of events over time were presented graphically in Kaplan-Meier curves.

Levels of circulating hormones in patients suffering from cancer at different disease stages were compared using the Mann-Whitney U test.

In order to determine the relationships between cytokines and hormones, we performed a multiple linear regression analysis. The variables age, gender and the inflammatory markers CRP, IL-6, haptoglobin, fibronectin and SAA were used as predictors for each hormone. Moreover, correlation with hsTnT was investigated.

For all tests, two-sided $\mathrm{p}$ values $<0.05$ were considered to indicate statistical significance.

\section{RESULTS}

\section{Baseline characteristics}

A total of 600 consecutive patients were enrolled in our prospective cohort study. In 45 patients, the diagnosis of cancer could not be confirmed, and they were, therefore, excluded from further analysis. The detailed baseline characteristics of our study population are displayed in table 1. A complete description of tumour entities is presented in table 2 . 
Table 1 Baseline characteristics of patients with newly diagnosed cancer $(n=555)$

Patients with cancer $(n=555)$

\begin{tabular}{|c|c|}
\hline Age, years (IQR) & $62(52-71)$ \\
\hline Male gender, $\mathrm{n}(\%)$ & $197(40 \%)$ \\
\hline BMI kg/m², (IQR) & $25.0(22.6-28.4)$ \\
\hline \multicolumn{2}{|l|}{ Comorbidities } \\
\hline Known CAD, n (\%) & $28(5 \%)$ \\
\hline Valvular disease, $\mathrm{n}(\%)$ & $8(1 \%)$ \\
\hline Heart failure, $\mathrm{n}(\%)$ & $38(7 \%)$ \\
\hline Stroke/TIA, n (\%) & $17(3 \%)$ \\
\hline PAD, n (\%) & $19(3 \%)$ \\
\hline Diabetes mellitus, $\mathrm{n}(\%)$ & $43(8 \%)$ \\
\hline Arterial hypertension, $\mathrm{n}(\%)$ & $250(45 \%)$ \\
\hline CKD, n (\%) & $31(6 \%)$ \\
\hline COPD, n (\%) & $113(20 \%)$ \\
\hline Currently smoking, n (\%) & $118(21 \%)$ \\
\hline Sinus rhythm, $\mathrm{n}(\%)$ & $509(96 \%)$ \\
\hline PM or ICD, n (\%) & $6(1 \%)$ \\
\hline \multicolumn{2}{|l|}{ Medication } \\
\hline Beta-blocker, n (\%) & $123(22 \%)$ \\
\hline ACE or ARB, $n(\%)$ & $166(31 \%)$ \\
\hline Aldosterone antagonist, $\mathrm{n}(\%)$ & $15(3 \%)$ \\
\hline Digitalis, n (\%) & $3(1 \%)$ \\
\hline ASS, n (\%) & $111(20 \%)$ \\
\hline Planned therapy* & $383(89 \%)$ \\
\hline Surgery, n (\%) & $318(75 \%)$ \\
\hline Anticancer chemotherapy, n (\%) & $298(70 \%)$ \\
\hline Hormone therapy, n (\%) & $66(16 \%)$ \\
\hline Radiation therapy, n (\%) & $146(34 \%)$ \\
\hline Antibody therapy, n (\%) & $80(19 \%)$ \\
\hline Bone marrow transplantation, $\mathrm{n}(\%)$ & $4(1 \%)$ \\
\hline Normal cardiac status, n (\%) & $408(75 \%)$ \\
\hline Serum creatinine, mg/dL (IQR) & $0.88(0.77-1.03)$ \\
\hline $\mathrm{GFR}, \mathrm{mL} / \mathrm{min} / 1.73 \mathrm{~m}^{2}$ (IQR) & $74.5(63.7-86.0)$ \\
\hline hsTnT, ng/mL (IQR) & $0.006(0.003-0.010)$ \\
\hline NT-proBNP, pg/mL (IQR) & $128(64-279)$ \\
\hline MR-proANP, pmol/L (IQR) & $66.5(47.2-107.3)$ \\
\hline MR-proADM, nmol/L (IQR) & $0.56(0.44-0.72)$ \\
\hline CT-proET-1, pmol/L (IQR) & $52.5(40.3-68.1)$ \\
\hline Copeptin, pmol/L (IQR) & $4.60(2.90-8.28)$ \\
\hline CRP, mg/L (IQR) & $0(0-1)$ \\
\hline IL-6, pg/mL (IQR) & $2(2-3)$ \\
\hline Fibronectin, mg/dL (IQR) & $42(35-48)$ \\
\hline Haptoglobin, mg/dL (IQR) & $137(93-201)$ \\
\hline SAA, $\mu \mathrm{g} / \mathrm{mL}(\mathrm{IQR})$ & $8(4-26)$ \\
\hline
\end{tabular}

Continuous variables are given as medians and IQR. Counts are given as numbers and percentages.

*Information about planned therapy was collected for all patients excluding those with myeloproliferative neoplasias.

ARB, angiotensin receptor blocker; ASS, acetyl salicylic acid; BMI, body mass index; $C A D$, coronary artery disease; $C K D$, chronic kidney disease; COPD, chronic obstructive pulmonary disease; CRP, C reactive protein; CT-proET-1, C-terminal pro endothelin-1; GFR, glomerular filtration rate; hsTnT, high-sensitive troponin T; ICD, implantable cardioverter defibrillator; IL-6, interleukin 6; MR-proADM, mid-regional pro-adrenomedullin; MR-proANP, mid-regional pro-atrial natriuretic peptide; NT-proBNP, N-terminal pro B-type natriuretic peptide; PAD, peripheral artery disease; $\mathrm{PM}$, pacemaker; SAA, serum amyloid $\mathrm{A}$; TIA, transitory ischaemic attack.

\section{Cox regression analysis}

One hundred and eighty-six patients (34\%) died during a median follow-up of 25 months (IQR 16-31). The crude and basic adjusted predictive values and the C-statistics for the
Table 2 Tumour entities and staging for patients with confirmed cancer $(n=555)$

Patients with cancer $(\mathrm{n}=555)$

\begin{tabular}{|c|c|}
\hline \multicolumn{2}{|l|}{ Tumour entity } \\
\hline Lung cancer, n (\%) & $61(11.0 \%)$ \\
\hline Breast cancer, $\mathrm{n}(\%)$ & $146(26.3 \%)$ \\
\hline Brain tumour, $\mathrm{n}(\%)$ & $23(4.1 \%)$ \\
\hline ENT tumour, $\mathrm{n}(\%)$ & $33(5.9 \%)$ \\
\hline Gastrointestinal tumours, n (\%) & $67(12.1 \%)$ \\
\hline Myelodysplastic malignancies, n (\%) & $68(12.3 \%)$ \\
\hline Myeloproliferative neoplasias, n (\%) & $99(17.8 \%)$ \\
\hline Oesophageal cancer, n (\%) & $11(2.0 \%)$ \\
\hline Testicular cancer, n (\%) & $2(0.4 \%)$ \\
\hline Neuroendocrine tumour, n (\%) & $11(2.0 \%)$ \\
\hline Sarcoma, n (\%) & $9(1.6 \%)$ \\
\hline Mesothelioma, n (\%) & $3(0.6 \%)$ \\
\hline Prostate cancer, n (\%) & $2(0.4 \%)$ \\
\hline Renal cell carcinoma, n (\%) & $4(0.7 \%)$ \\
\hline Thymoma, n (\%) & $1(0.2 \%)$ \\
\hline Skin cancer, n (\%) & $2(0.4 \%)$ \\
\hline Urogenital tumours, n (\%) & $2(0.4 \%)$ \\
\hline Oral cancer, n (\%) & $1(0.2 \%)$ \\
\hline Other, n (\%) & $10(1.8 \%)$ \\
\hline \multicolumn{2}{|l|}{ Tumour stage } \\
\hline Stage $1, \mathrm{n}(\%)$ & $93(16.7 \%)$ \\
\hline Stage $2, \mathrm{n}(\%)$ & $49(8.8 \%)$ \\
\hline Stage $3, \mathrm{n}(\%)$ & $108(19.5 \%)$ \\
\hline Stage $4, n(\%)$ & $177(31.9 \%)$ \\
\hline No staging, $\mathrm{n}(\%)$ & $128(23.1 \%)$ \\
\hline
\end{tabular}

Counts are given as numbers and percentages.

Myelodysplastic malignancies: haematological malignancies with abnormal differentiation of myeloid or lymphoid cell lines (eg, AML, acute myeloid leukemia; ALL, acute lymphoid leukemia; lymphomas, multiple myeloma); myeloproliferative neoplasias: haematological neoplasias with normal cell differentiation (eg, essential thrombocytosis, polycythaemia vera, myelofibrosis).

ENT, ear, nose, throat.

hormones and hsTnT are displayed in table 3. In the basic model, all included variables except GFR and cardiac status were significant adverse risk factors for mortality, resulting in an overall C-statistic of 0.79 .

In the univariate analysis, NT-proBNP, MR-proANP, MR-proADM, CT-proET-1 and copeptin were significant predictors of mortality. After adjustment for the basic model, an independent significant predictive value could be established for all variables. NT-proBNP, MR-proANP and MR-proADM revealed the strongest predictive values $(p<0.001$ for NT-proBNP and MR-proADM, $p<0.01$ for MR-proANP). In addition, the cardiac marker hsTnT was similarly associated with mortality. When hsTnT was included in the basic model, the direct association with mortality remained significant for NT-proBNP $(p=0.013)$, MR-proADM $(p<0.001)$, CT-proET-1 $(p<0.001)$ and copeptin $(p=0.021)$, highlighting the independent impact of these hormones in cancer.

Kaplan-Meier analysis confirmed the high discriminatory power of NT-proBNP, MR-proADM, CT-proET-1 and hsTnT with estimated survival rates of $67 \%$ and $49 \%(\mathrm{p}<0.001)$ at a cut-off value of $125 \mathrm{pg} / \mathrm{mL}$ for NT-proBNP, $71 \%$ and $50 \%$ $(\mathrm{p}<0.001)$ at a cut-off value of $0.49 \mathrm{nmol} / \mathrm{L}$ for MR-proADM, $65 \%$ and $38 \%(\mathrm{p}<0.001)$ at a cut-off value of $66.6 \mathrm{pmol} / \mathrm{L}$ for CT-proET-1 and $69 \%$ and $48 \%(p<0.001)$ at a cut-off value of $0.005 \mathrm{ng} / \mathrm{mL}$ for hsTnT, respectively, shown in figure 1 . 
Table 3 Unadjusted and adjusted effects on mortality

\begin{tabular}{|c|c|c|c|c|c|c|c|c|c|c|}
\hline Variables & IQR & $\begin{array}{l}\text { Crude HR } \\
(95 \% \mathrm{CI})\end{array}$ & p Value & C-statistic & $\begin{array}{l}\text { Adj. HR* } \\
(95 \% \mathrm{Cl})\end{array}$ & p Value & C-statistic & $\begin{array}{l}\text { Adj. HRt } \\
(95 \% \mathrm{Cl})\end{array}$ & p Value & C-statistic \\
\hline hsTnT, ng/mL & 0.007 & 1.27 (1.20 to 1.34$)$ & $<0.001$ & 0.65 & 1.21 (1.13 to 1.32$)$ & $<0.001$ & 0.80 & - & - & - \\
\hline In(NT-proBNP), pg/mL & 1.465 & 1.56 (1.34 to 1.83$)$ & $<0.001$ & 0.63 & 1.54 (1.24 to 1.90$)$ & $<0.001$ & 0.80 & 1.33 (1.06 to 1.68$)$ & 0.013 & 0.81 \\
\hline In(MR-proANP), pmol/L & 0.821 & $1.45(1.21$ to 1.72$)$ & $<0.001$ & 0.59 & 1.40 (1.10 to 1.79$)$ & 0.006 & 0.80 & 1.21 (0.94 to 1.55$)$ & 0.142 & 0.81 \\
\hline MR-proADM, nmol/L & 0.280 & 1.23 (1.20 to 1.36$)$ & $<0.001$ & 0.65 & 1.31 (1.19 to 1.44$)$ & $<0.001$ & 0.81 & 1.24 (1.12 to 1.38$)$ & $<0.001$ & 0.81 \\
\hline CT-proET-1, pmol/L & 27.858 & 1.27 (1.20 to 1.35$)$ & $<0.001$ & 0.61 & 1.21 (1.14 to 1.30$)$ & $<0.001$ & 0.80 & 1.19 (1.11 to 1.28$)$ & $<0.001$ & 0.81 \\
\hline In(copeptin), pmol/L & 1.049 & 1.49 (1.28 to 1.75$)$ & $<0.001$ & 0.60 & 1.22 (1.04 to 1.42$)$ & 0.014 & 0.79 & 1.21 (1.03 to 1.42$)$ & 0.021 & 0.80 \\
\hline
\end{tabular}

Cox proportional hazard models for cardiovascular hormones are shown. HRs refer to a 1-IQR increase in continuous variables. HRs are adjusted (adj.) for the null model built from all variables selected by bootstrapping, that is, for age, tumour entity (tumours of categories 1, 2, 3, 6, 10 and 18) and condition of tumour stage 4 and additionally for GFR and cardiac status. The discriminatory power of the respective variables was assessed using Harrell's c-index.

${ }^{*} \mathrm{HR}$ adjusted to age, tumour stage 4, tumour entity, cardiac status and GFR.

THR adjusted to age, tumour stage 4, tumour entity, cardiac status, GFR and hsTnT.

Fonts in bold indicate statistical significance $(p<0.05)$

CT-proET-1, C-terminal pro endothelin-1; GFR, glomerular filtration rate; hsTnT, high-sensitive troponin T; MR-proADM, mid-regional pro-adrenomedullin; MR-proANP, mid-regional pro-atrial natriuretic peptide; NT-proBNP, N-terminal pro B-type natriuretic peptide.

\section{Hormones and hsTnT at different cancer stages}

NT-proBNP, MR-proANP, MR-proADM, CT-pro-ET-1, copeptin and hsTnT levels were elevated in patients with cancer, and increased with advancing tumour stage (table 4). IL-6, CRP, haptoglobin and SAA were similarly increased in patients with stage 4 disease compared with less advanced stages $(\mathrm{p}<0.05$ for all).

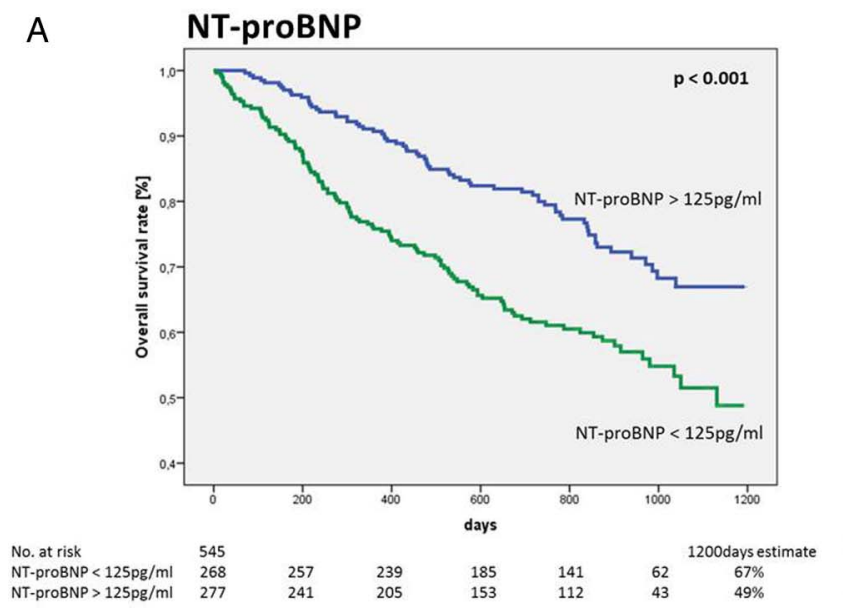

C

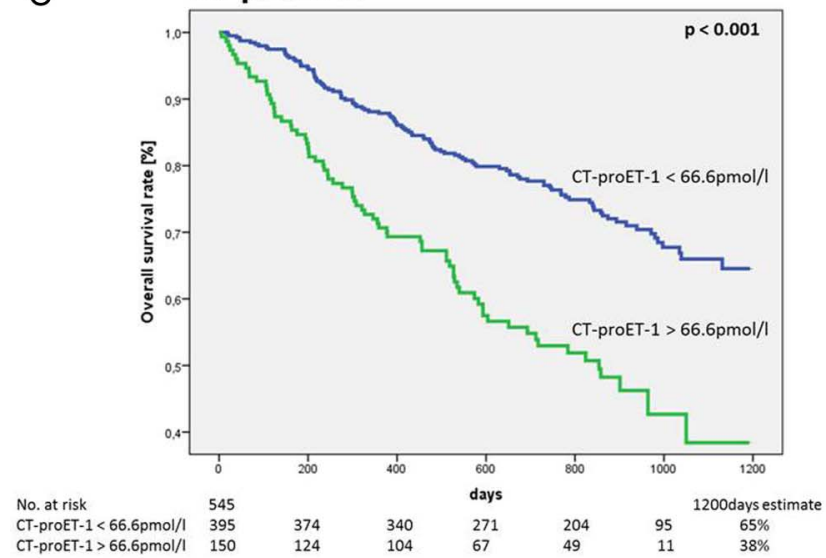

\section{Correlation between cytokines and hormones/hsTnT}

In the multiple linear regression model, direct correlation between the proinflammatory cytokine IL- 6 and the inflammatory marker CRP could be shown for MR-proADM $(p<0.05$ for IL- 6 and $p<0.001$ for CRP) with a corrected $R^{2}$ value of 0.41 , NT-proBNP $(\mathrm{p}<0.05$ for IL-6 and $\mathrm{p}<0.001$ for CRP) with a corrected $\mathrm{R}^{2}$ value of 0.31 and MR-proANP $(\mathrm{p}<0.001$

B

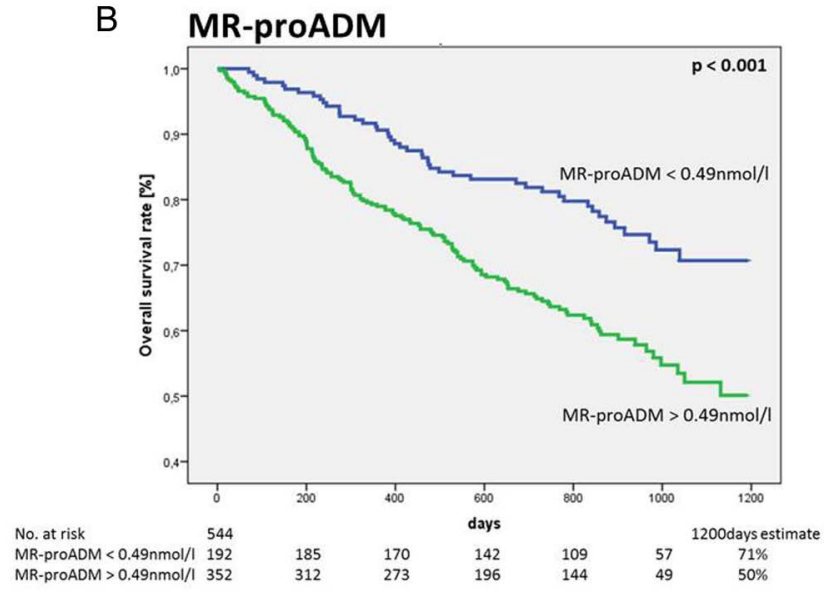

D

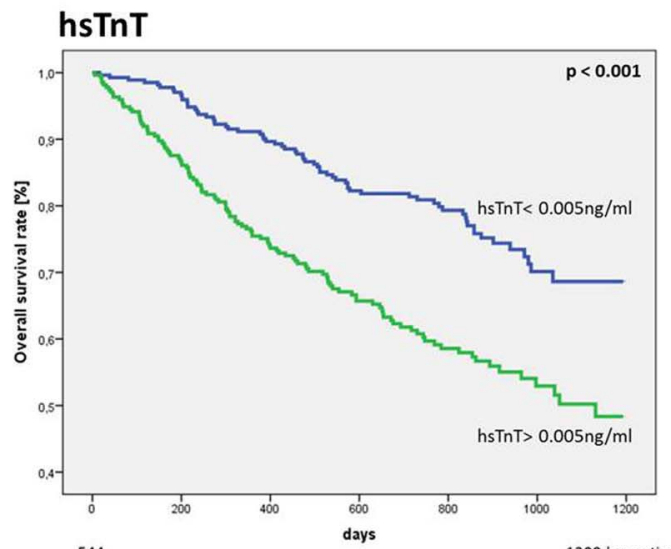

No. at risk hSTTT $<0.005 \mathrm{ng} / \mathrm{ml}$

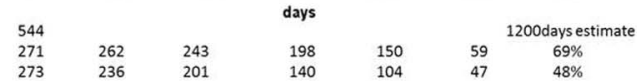

Figure 1 Kaplan-Meier estimates of all-cause mortality for (A) N-terminal pro B-type natriuretic peptide (NT-proBNP) with a cut-off value of $125 \mathrm{pg} / \mathrm{mL}$, (B) mid-regional pro-adrenomedullin (MR-proADM) with a cut-off value at $0.49 \mathrm{nmol} / \mathrm{L}$, (C) CT-proET-1 with a cut-off value of $66.6 \mathrm{pmol} / \mathrm{L}$ and (D) hsTnT with a cut-off value of $0.005 \mathrm{ng} / \mathrm{mL}$ for patients with newly diagnosed tumour disease $(p<0.001$ between two groups for NT-proBNP, CT-proET-1, MR-proADM and hsTnT, log-rank test). 
Table 4 Cardiovascular hormones, hsTnT and cytokines at different tumour stages

\begin{tabular}{|c|c|c|c|c|c|}
\hline Variables & Stage $1(n=96)$ & Stage $2(n=50)$ & Stage $3(n=108)$ & Stage $4(n=183)$ & $\begin{array}{l}p \text { Value tumour stages } \\
1,2,3 \text { vs tumour stage } 4\end{array}$ \\
\hline hsTnT, ng/mL (IQR) & 0.005 (0.003 to 0.009$)$ & 0.004 (0.003 to 0.007$)$ & 0.006 (0.003 to 0.011$)$ & 0.007 (0.004 to 0.012 ) & $<0.001$ \\
\hline NT-proBNP, pg/mL (IQR) & 103 (48 to 190$)$ & 105 (58 to 191$)$ & 118 (73 to 257 ) & 168 (74 to 377 ) & 0.002 \\
\hline MR-proANP, pmol/L (IQR) & 56.2 (45.3 to 83.8 ) & 61.1 (51.4 to 71.2 ) & 59.4 (41.6 to 99.9$)$ & 67.7 (47.4 to 113.5$)$ & 0.016 \\
\hline MR-proADM, nmol/L (IQR) & $0.49(0.41$ to 0.65$)$ & 0.51 (0.44 to 0.63$)$ & $0.53(0.41$ to 0.68$)$ & 0.62 (0.48 to 0.82 ) & $<0.001$ \\
\hline CT-proET-1, pmol/L (IQR) & 45.8 (39.0 to 57.4$)$ & 45.8 (38.3 to 58.6$)$ & 48.6 (38.0 to 59.1$)$ & 57.2 (42.4 to 74.1$)$ & $<0.001$ \\
\hline Copeptin, pmol/L (IQR) & $3.7(2.5$ to 6.9$)$ & $3.5(2.5$ to 5.0$)$ & 3.9 (2.6 to 6.6$)$ & $5.4(3.2$ to 10.6$)$ & $<0.001$ \\
\hline CRP, mg/L (IQR) & $0(0$ to 1$)$ & $0(0$ to 1$)$ & $0(0$ to 1$)$ & 1 (0 to 2$)$ & $<0.001$ \\
\hline IL-6, pg/mL (IQR) & 2 (2 to 2 ) & 2 (2 to 3$)$ & 2 (2 to 3$)$ & 2 (2 to 3$)$ & 0.011 \\
\hline Fibronectin, mg/dL (IQR) & 43 (36 to 47$)$ & 43 (34 to 48 ) & 43 (36 to 49$)$ & 42 (35 to 49 ) & 0.504 \\
\hline Haptoglobin, mg/dL (IQR) & 119 (93 to 171) & 144 (108 to 214$)$ & 146 (96 to 214$)$ & 178 (129 to 249$)$ & $<0.001$ \\
\hline Serum amyloid, $\mu \mathrm{g} / \mathrm{mL}$ (IQR) & $7(4$ to 17$)$ & 8 (4 to 25$)$ & $8(5$ to 19$)$ & $17(6$ to 51$)$ & $<0.001$ \\
\hline
\end{tabular}

Variables are displayed as median and IQR. Difference between patients with tumour stages 1, 2, 3 versus patients with tumour stage 4 was calculated by the means of the Mann-Whitney U test.

Fonts in bold indicate statistical significance $(p<0.05)$.

CRP, C reactive protein; CT-proET-1, C-terminal pro endothelin-1; hsTnT, high-sensitive troponin T; IL-6, interleukin 6; MR-proADM, mid-regional pro-adrenomedullin; MR-proANP, mid-regional pro-atrial natriuretic peptide; NT-proBNP, N-terminal pro B-type natriuretic peptide.

for both IL-6 and CRP, respectively) with corrected $\mathrm{R}^{2}$ value of 0.43. For hsTnT, the association was significant with CRP $(p>0.001)$, with a corrected $R^{2}$ value of 0.60 .

\section{DISCUSSION}

Elevated levels of cardiovascular peptides and hsTnT have been reported in patients with cancer. However, the underlying mechanism remains speculative since a clinical manifestation of cardiac disease could not be confirmed. To the best of our knowledge, this is the first study to investigate the association between circulating cardiovascular functional peptides (ie, NT-proBNP, MR-proANP, MR-proADM, CT-proET-1 and copeptin) and the cardiac morphological marker hsTnT with allcause mortality in an unselected population with cancer prior to the induction of anticancer therapy. We demonstrated that these markers are systematically elevated in these patients, up to 100 -fold of the upper reference limit, and that they are likewise related to long-term mortality independently of age, gender, tumour entity, tumour stage and manifest cardiac disease at first clinical presentation. Additionally, the hormones NT-proBNP, MR-proANP and MR-proADM significantly correlated with the proinflammatory cytokine IL-6 and the inflammatory marker CRP, as well as with hsTnT as an indicator of myocardial damage.

\section{Association between cardiovascular peptides, cytokines and cancer}

Alterations to the vasoactive peptides ADM, ET-1 and AVP are involved in the development and progression of cardiac diseases, especially in heart failure. ${ }^{5}$ In addition to their role in cardiac disease, ADM, ET-1 and AVP have been implicated in the process of tumour progression through regulation of angiogenesis. ${ }^{719}$ Furthermore, natriuretic peptides have been identified as powerful predictors of mortality in various cardiovascular diseases. ${ }^{4} 6$ Although regarded as cardiac hormones, recent data suggest that the biological functions of BNP and ANP are restricted to cardiovascular haemodynamics, and play an important role in cancer by regulating cell growth, alleviating oxidant-induced cell damage, inflammatory processes as well as cancer cachexia. ${ }^{20}$

Current cancer research focuses predominantly on the regulation of the tumour microenvironment by cytokines ${ }^{21} 22$ or
ADM, ET-1 and AVP. 192324 The effect of ADM on angiogenesis and proliferation has already been proposed as a novel therapeutic target in different cancer types. ${ }^{7}$ In analogy, the cytokine hypothesis in heart failure has been established as a complementary concept suggesting that an uncontrolled local activation of cytokines like IL- 6 can trigger neurohumoral activation, fibrotic processes and apoptosis. ${ }^{25} 26$ In contrast to cancer, studies have mainly focused on circulating cardiovascular hormones in cardiac disease. However, little is known about the interaction and progression of both diseases when common hormones become systemically active in patients with cancer.

Our data reveal a central role for NT-proBNP, MR-proANP, MR-proADM, CT-proET-1 and copeptin in the prediction of overall survival in patients with cancer. Furthermore, we were able to confirm a significant correlation between the proinflammatory cytokine IL-6 and the inflammatory marker CRP and the hormones NT-proBNP, MR-proANP and MR-proADM. Whether the effect on mortality is primarily due to a detrimental local influence on the tumour microenvironment or is induced by systemic cardiovascular dysregulation cannot be determined.

\section{Cardiac involvement in cancer}

Occurrence of cardiac disease induced by anticancer treatment is a well-studied clinical situation. However, little is known about cancer-induced cardiac injury independent of anticancer therapy. Associations between increased NT-proBNP and cancer as well as the presence of extremely high peptide levels for specific tumour entities have been previously reported. ${ }^{10} \quad 11 \quad 14$ Nevertheless, these findings could not be correlated with evidence of morphological myocardial dysfunction. ${ }^{10}{ }^{11}$ Recent studies also demonstrated an association between elevated TnT levels in patients with cancer without evidence of cardiac disease, but an adverse effect on mortality, indicating myocyte cell injury. ${ }^{15} 16$ In accordance with these results, our data show an elevation of all investigated vasoactive peptides and hsTnT as well as a good correlation between the hormones and hsTnT, strengthening this hypothesis. The highly predictive value of cardiovascular peptides may rely on their ability to detect subclinical functional impairment of the cardiovascular system caused by malignant disease prior to detectable changes in routine echocardiographic examinations. 
Table 5 Cardiovascular hormones and hsTnT in myelodysplastic malignancies and solid tumours

\begin{tabular}{lccc}
\hline & Myelodysplastic malignancies $(\mathbf{n}=68)$ & Solid tumours $(\mathbf{n}=\mathbf{3 8 8})$ & $\mathrm{p}$ Value \\
\hline hsTnT, ng/mL (IQR) & $0.006(0.003-0.011)$ & $0.006(0.003-0.011)$ & 0.962 \\
NT-proBNP, pg/mL (IQR) & $153(59-352)$ & $125(66-268)$ & 0.520 \\
MR-proANP, pmol/L (IQR) & $69.0(50.1-117.6)$ & $62.4(44.4-100.5)$ & 0.306 \\
MR-proADM, nmol/L (IQR) & $0.61(0.44-0.90)$ & $0.54(0.43-0.72)$ & 0.129 \\
CT-proET-1, pmol/L (IQR) & $50.1(39.3-67.6)$ & $50.0(39.4-66.1)$ & 0.797 \\
Copeptin, pmol/L (IQR) & $4.8(3.0-9.3)$ & $4.2(2.7-7.9)$ & 0.226 \\
\hline
\end{tabular}

Variables are displayed as median and IQR. Differences were calculated by the means of the Mann-Whitney U test.

CT-proET-1, C-terminal pro endothelin-1; hsTnT, high-sensitive troponin T; MR-proADM, mid-regional pro-adrenomedullin; MR-proANP, mid-regional pro-atrial natriuretic peptide; NT-proBNP, N-terminal pro B-type natriuretic peptide

A potential contributor to cardiac impairment in cancer could be cardiac cachexia, which often accompanies advanced cancer states. $^{27} 28$ In a rodent hepatoma model, elevated levels of BNP were attributed to cardiac cachexia associated with cardiac remodelling, deterioration of cardiac function and death. ${ }^{18}$ Interestingly, the treatment of rodents with spironolactone and beta-blocker, targeting established neurohumoral pathways of heart failure, succeeded in reducing mortality, ${ }^{18}$ but could not yet successfully be translated into clinical practice.

\section{Clinical implications}

Conventional chemotherapeutics are associated with permanent (type I) or transient (type II) myocardial cell injury, thereby potentially affecting the quality of life and overall survival of patients with cancer. $^{29}$ Along with other parameters, the measure of cardiac biomarkers such as NT-proBNP, TnT or ultrasensitive cardiac troponin I has been suggested to reflect and help monitor cardiac function during chemotherapy. ${ }^{29} 30$ Re-evaluation and cessation of chemotherapy may be considered in patients with increasing levels of the aforementioned biomarkers during cardiotoxic anticancer therapy. ${ }^{29}$ However, our results indicate that higher initial levels of cardiovascular markers should be expected in patients with cancer. Moreover, elevated NT-proBNP levels, especially in the absence of previous cardiovascular disease, should be interpreted with caution, since an increased NT-proBNP level might reflect a more malignant disease, thereby identifying patients with a more critical need for diagnosis and therapy.

We expect that assessing subclinical myocardial injury detected by hsTnT can provide important additional information to guide cardiotoxic anticancer therapy, indicating cardiac damage prior to reduced LV function becoming apparent in echocardiography. This might be relevant for some therapies classified as type II cardiotoxic substances, which change to type I substances in the presence of pre-existing cardiac disease. ${ }^{29}$

Furthermore, vasoactive peptides might serve as novel therapeutic targets as recently proposed for ADM. ${ }^{7}$ In light of our data, more focus should be placed on systemic rather than local effects of vasoactive peptides.

Additionally, small experimental studies have already successfully demonstrated a positive effect of heart failure-specific therapy in patients with cancer. ${ }^{18}$ Our results provide intellectual support for this approach beyond the prevention of known cardiotoxic drug-related effects.

\section{Limitations}

One potential limitation of this study is the unselective enrolment of patients, including various types of cancer. Neuroendocrine tumours specifically could behave differently, but those only accounts for $2 \%$ of our study population, and do not have any influence on our results. Moreover, our data show a comparable predictive value for hsTnT, MR-proADM and CT-proET-1 for solid and myelodysplastic malignancies, but a significant interaction between these groups and MR-proADM as well as CT-proET-1 with regard to the primary outcome (tables 5 and 6). Nevertheless, we intended to investigate peptide activation as a general phenomenon in cancer, without focusing on distinct tumour entities. Further studies might reveal more differences between various types of cancer. Also, laboratory measurements have been performed only at a single time point prior to initiation of systemic anticancer therapy, and studies with serial measurements throughout disease progression might provide additional insights. Finally, while our endpoint is all-cause mortality, precise information about the percentage of

Table 6 Unadjusted effects on mortality and interaction for myelodysplastic malignancies versus solid tumours

\begin{tabular}{|c|c|c|c|c|c|c|}
\hline \multirow[b]{2}{*}{ Variables } & \multirow[b]{2}{*}{ IQR } & \multicolumn{2}{|c|}{ Myelodysplastic malignancies $(n=68)$} & \multicolumn{2}{|c|}{ Solid tumours $(n=388)$} & \multirow{2}{*}{$\begin{array}{l}p \text { Value } \\
\text { Interaction }\end{array}$} \\
\hline & & Crude HR (95\% CI) & $\mathrm{p}$ Value & Crude HR (95\% Cl) & $\mathrm{p}$ Value & \\
\hline hsTnT, ng/mL & 0.007 & 1.24 (1.08 to 1.42$)$ & 0.001 & 1.35 (1.25 to 1.44$)$ & $<0.001$ & 0.238 \\
\hline $\ln (\mathrm{NT}-\mathrm{proBNP}), \mathrm{pg} / \mathrm{mL}$ & 1.465 & $1.58(0.93$ to 2.69$)$ & 0.093 & 1.66 (1.39 to 1.98$)$ & $<0.001$ & 0.140 \\
\hline In(MR-proANP), pmol/L & 0.821 & 1.27 (0.64 to 2.49$)$ & 0.495 & 1.59 (1.33 to 1.91$)$ & $<0.001$ & 0.242 \\
\hline MR-proADM, nmol/L & 0.280 & $2.10(1.42$ to 3.10$)$ & $<0.001$ & 1.24 (1.16 to 1.32$)$ & $<0.001$ & 0.001 \\
\hline CT-proET-1, pmol/L & 27.858 & 1.74 (1.09 to 2.76$)$ & 0.020 & 1.25 (1.18 to 1.33$)$ & $<0.001$ & 0.025 \\
\hline In(copeptin), pmol/L & 1.049 & $1.55(0.73$ to 3.29$)$ & 0.251 & $1.57(1.36$ to 1.83$)$ & $<0.001$ & 0.177 \\
\hline
\end{tabular}

Cox proportional hazard model of cardiovascular hormones and hsTnT for haematological myelodysplastic and solid tumours and the $\mathrm{p}$ value for interaction between the groups are shown. HRs refer to a 1-IQR increase in continuous variables.

Fonts in bold indicate statistical significance $(p<0.05)$.

CT-proET-1, C-terminal pro endothelin-1; hsTnT, high-sensitive troponin T; MR-proADM, mid-regional pro-adrenomedullin; MR-proANP, mid-regional pro-atrial natriuretic peptide; NT-proBNP, N-terminal pro B-type natriuretic peptide. 
cardiovascular-related death would certainly be of important clinical interest. Since post-hoc interpretations of certifications of death are not reliable, the development of a cardiac disease during cancer progression should be documented in longitudinal studies in the future.

\section{CONCLUSION}

In conclusion, cardiovascular functional peptides like NT-proBNP, MR-proANP, MR-proADM, CT-pro-ET-1 and copeptin as well as the cardiac morphological marker hsTnT are systemically elevated in patients with cancer prior to cardiotoxic therapy and in the absence of concurrent infection. All of these markers are strongly related to mortality, implying a direct association with disease progression.

\section{Key messages}

\section{What is already known on this subject?}

Patients with cancer may display elevated levels of B-type natriuretic peptide (BNP) and high-sensitive troponin T (hsTnT) in the absence of any clinical manifestation of cardiac disease.

\section{What might this study add?}

This study shows that circulating cardiovascular hormones such as N-terminal pro BNP, mid-regional pro-atrial natriuretic peptide, mid-regional pro-adrenomedullin, C-terminal pro-endothelin-1 and copeptin as well as hsTnT are related to cancer disease progression and severity suggesting the presence of subclinical functional and morphological myocardial damage.

\section{How might this impact on clinical practice?}

The results provide intellectual support of heart failure therapy in patients with cancer beyond the current focus on preventing therapy-related cardiotoxic side effects. Furthermore, our study provides insight into the expected levels of cardiovascular hormones in a non-selected population of patients with cancer.

Acknowledgements We thank Maysa Sarhan for editing the manuscript and Isabella Brodnjak for her valuable help with data collection.

Contributors Conception or design of the work: Martin Hülsmann (MH), Richard Pacher (RP) and NP. Analysis or interpretation of data for the work: Martin Hülsmann (MH), Richard Pacher (RP), GS, NP, MR, HG, GGS, Michael Hejna (MH), WK, SZ-M, CM, GK, LA, SS, BP, WS, Robert Pirker (RP), JD and CZ. Drafting and critically revising the manuscript: Martin Hülsmann (MH), NP, SN, CA and GG.

Funding This study was supported by an unrestricted grant of Thermo Fisher.

Competing interests None declared.

Ethics approval Ethics Committee of The Medical University of Vienna.

Provenance and peer review Not commissioned; externally peer reviewed.

\section{REFERENCES}

1 Coleman MP. Cancer survival: global surveillance will stimulate health policy and improve equity. Lancet 2014;383:564-73.

2 Goubran HA, Kotb RR, Stakiw J, et al. Regulation of tumor growth and metastasis: the role of tumor microenvironment. Cancer Growth Metastasis 2014;7:9-18.
3 Wrigley BJ, Lip GY, Shantsila E. The role of monocytes and inflammation in the pathophysiology of heart failure. Eur J Heart Fail 2011;13:1161-71.

4 Hulsmann M, Berger R, Sturm B, et al. Prediction of outcome by neurohumoral activation, the six-minute walk test and the Minnesota Living with Heart Failure Questionnaire in an outpatient cohort with congestive heart failure. Eur Heart J 2002;23:886-91.

5 Adlbrecht C, Hulsmann M, Strunk G, et al. Prognostic value of plasma midregional pro-adrenomedullin and C-terminal-pro-endothelin-1 in chronic heart failure outpatients. Eur J Heart Fail 2009;11:361-6.

6 Khan SQ, Dhillon O, Kelly D, et al. Plasma N-terminal B-Type natriuretic peptide as an indicator of long-term survival after acute myocardial infarction: comparison with plasma midregional pro-atrial natriuretic peptide: the LAMP (Leicester Acute Myocardial Infarction Peptide) study. J Am Coll Cardiol 2008;51:1857-64.

7 Nikitenko LL, Fox SB, Kehoe S, et al. Adrenomedullin and tumour angiogenesis. Br J Cancer 2006;94:1-7.

8 Morawiec B, Kawecki D. Copeptin: a new marker in cardiology. J Cardiovasc Med (Hagerstown) 2013;14:19-25.

9 Nelson J, Bagnato A, Battistini B, et al. The endothelin axis: emerging role in cancer. Nat Rev Cancer 2003;3:110-16.

10 Law C, Glover C, Benson K, et al. Extremely high brain natriuretic peptide does not reflect the severity of heart failure. Congest Heart Fail 2010;16:221-5.

11 Burjonroppa SC, Tong AT, Xiao LC, et al. Cancer patients with markedly elevated B-type natriuretic peptide may not have volume overload. Am J Clin Oncol 2007;30:287-93.

12 Wigle DA, Campling BG, Sarda IR, et al. ANP secretion from small cell lung cancer cell lines: a potential model of ANP release. Am J Physiol 1995;268:H1869-74.

13 Ohsaki Y, Gross AJ, Le PT, et al. Human small cell lung cancer cells produce brain natriuretic peptide. Oncology 1999;56:155-9.

14 Popat J, Rivero A, Pratap P, et al. What is causing extremely elevated amino terminal brain natriuretic peptide in cancer patients? Congest Heart Fail 2013;19:143-8

15 Lim E, Li Choy L, Flaks L, et al. Detected troponin elevation is associated with high early mortality after lung resection for cancer. J Cardiothorac Surg 2006;1:37.

16 Danese E, Montagnana M, Giudici S, et al. Highly-sensitive troponin I is increased in patients with gynecological cancers. Clin Biochem 2013;46:1135-8.

17 von Haehling S, Doehner W, Anker SD. Nutrition, metabolism, and the complex pathophysiology of cachexia in chronic heart failure. Cardiovasc Res 2007;73:298-309.

18 Springer J, Tschirner A, Haghikia A, et al. Prevention of liver cancer cachexia-induced cardiac wasting and heart failure. Eur Heart J 2014;35:932-41.

19 Pedram A, Razandi M, Hu RM, et al. Vasoactive peptides modulate vascular endothelial cell growth factor production and endothelial cell proliferation and invasion. J Biol Chem 1997;272:17097-103.

20 De Vito P. Atrial natriuretic peptide: an old hormone or a new cytokine? Peptides 2014;58:108-16.

21 Lippitz BE. Cytokine patterns in patients with cancer: a systematic review. Lancet Oncol 2013;14:e218-28.

22 Pine SR, Mechanic LE, Enewold L, et al. Increased levels of circulating interleukin 6, interleukin 8, C-reactive protein, and risk of lung cancer. J Nat/ Cancer Inst 2011;103:1112-22.

23 Kocemba KA, van Andel $\mathrm{H}$, de Haan-Kramer $\mathrm{A}$, et al. The hypoxia target adrenomedullin is aberrantly expressed in multiple myeloma and promotes angiogenesis. Leukemia 2013;27:1729-37.

24 Knowles J, Loizidou M, Taylor I. Endothelin-1 and angiogenesis in cancer. Curr Vasc Pharmacol 2005;3:309-14.

25 Gwechenberger M, Hulsmann M, Berger R, et al. Interleukin-6 and B-type natriuretic peptide are independent predictors for worsening of heart failure in patients with progressive congestive heart failure. J Heart Lung Transplant 2004;23:839-44.

26 Prabhu SD. Cytokine-induced modulation of cardiac function. Circ Res 2004;95:1140-53.

27 Tisdale MJ. Biology of cachexia. J Natl Cancer Inst 1997;89:1763-73.

28 von Haehling S, Lainscak M, Springer J, et al. Cardiac cachexia: a systematic overview. Pharmacol Ther 2009;121:227-52.

29 Suter TM, Ewer MS. Cancer drugs and the heart: importance and management. Eur Heart J 2013;34:1102-11.

30 Moreno R, Costa RG, Mazar RC, et al. Evaluation of ultrasensitive cardiac Troponin I levels in patients with breast cancer during chemotherapy. Clin Chim Acta 2014;428:70-1. 